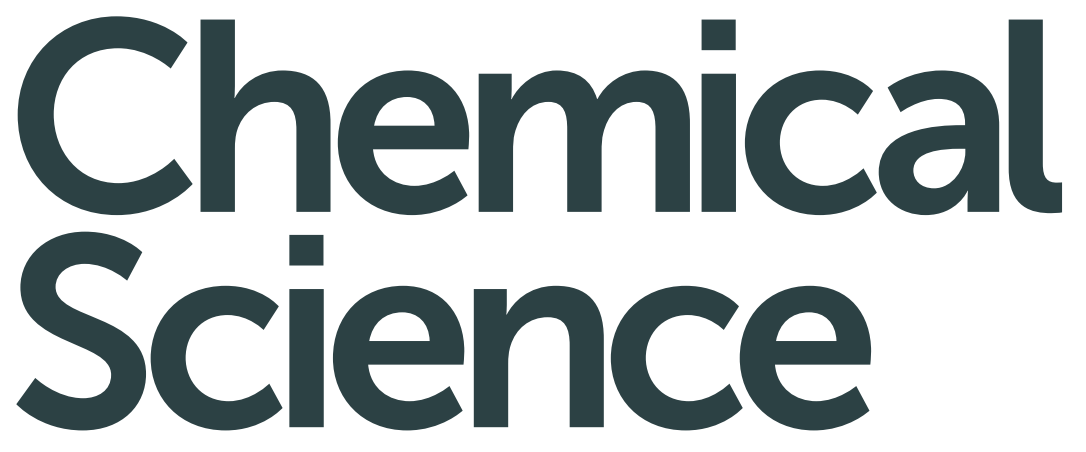

rsc.li/chemical-science

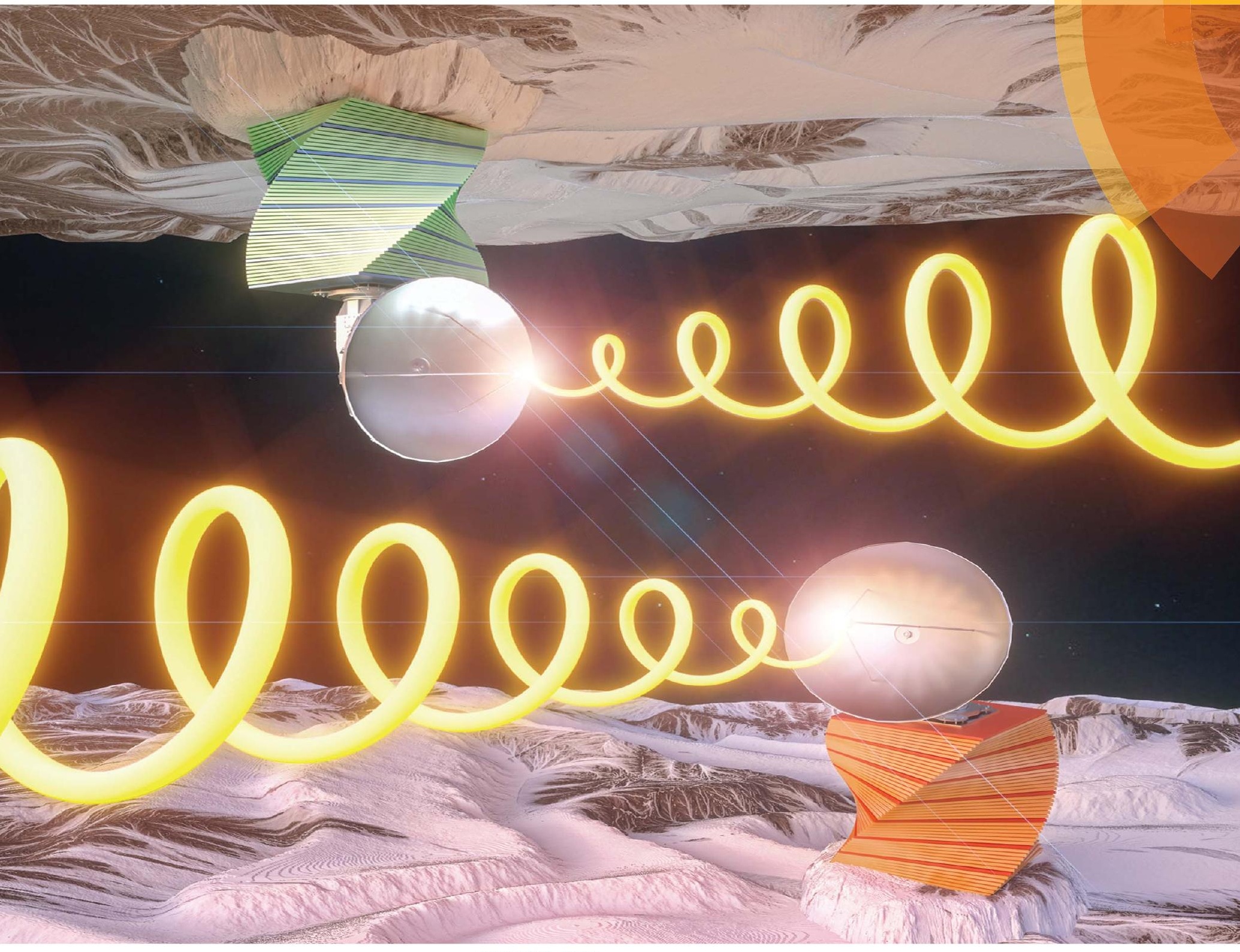

ISSN 2041-6539

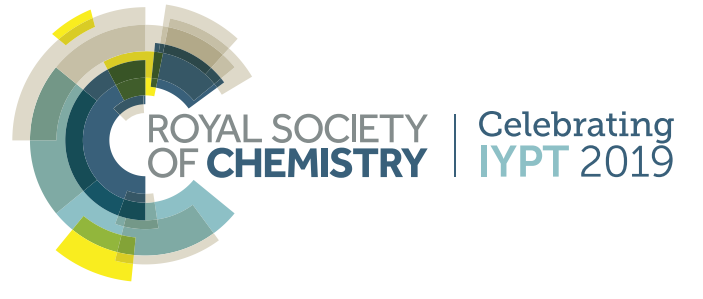

EDGE ARTICLE

Youngmin You et al

Amplified circularly polarized phosphorescence

from co-assemblies of platinum(II) complexes 
Check for updates

Cite this: Chem. Sci., 2019, 10, 1294

๑ All publication charges for this article have been paid for by the Royal Society of Chemistry

Received 10th October 2018

Accepted 29th November 2018

DOI: $10.1039 / \mathrm{c} 8 \mathrm{sc} 04509 \mathrm{~g}$

rsc.li/chemical-science

\title{
Amplified circularly polarized phosphorescence from co-assemblies of platinum(II) complexes $\uparrow$
}

\author{
Gyurim Park, ${ }^{a}$ Hyungchae Kim, ${ }^{\mathrm{b}}$ Hoichang Yang, (D) ${ }^{\mathrm{c}}$ Kyung Ryoul Park, ${ }^{\mathrm{b}}$ Inho Song, ${ }^{\mathrm{de}}$ \\ Joon Hak Oh, (D) Changsoon Kim (iD ${ }^{\mathrm{b}}$ and Youngmin You (DD ${ }^{\text {*a }}$
}

\begin{abstract}
Molecules capable of producing zero-field circularly polarized phosphorescence (CPP) are highly valuable for chiroptoelectronic applications that rely on triplet exciton. However, the paucity of tractable molecular design rules for obtaining CPP emission has inhibited full utilization. We report amplification of CPP by the formation of helical co-assemblies consisting of achiral square planar cycloplatinated complexes and small fractions of homochiral cycloplatinated complexes. The latter has a unique Pfeiffer effect during the formation of superhelical co-assemblies, enabling versatile chiroptical control. Large dissymmetry factors in electronic absorption $\left(g_{\mathrm{abs}}, 0.020\right)$ and phosphorescence emission $\left(g_{\text {lum }}, 0.064\right)$ are observed from the co-assemblies. These values are two orders of magnitude improved relative to those of individual molecules. In addition, photoluminescence quantum yields (PLQY) also increase by a factor of ten. Our structural, photophysical, and quantum chemical investigations reveal that the chiroptical amplification is attributable to utilization of both the magnetically allowed electronic transition and asymmetric coupling of excitons. The strategy overcomes the trade-off between $g_{\text {lum }}$ and PLQY which has frequently been found for previous molecular emitters of circularly polarized luminescence. It is anticipated that our study will provide new insight into the future research for the exploitation of the full potential of CPP.
\end{abstract}

\section{Introduction}

Circularly polarized luminescence (CPL) refers to photoemission that is enriched with light having a polarization axis

${ }^{a}$ Division of Chemical Engineering and Materials Science, Ewha Womans University, Seoul 03760, The Republic of Korea. E-mail: odds2@ewha.ac.kr

${ }^{b}$ Graduate School of Convergence Science and Technology, Inter-University Semiconductor Research Center, Seoul National University, Seoul 08826, The Republic of Korea

'Department of Chemical Engineering, Inha University, Incheon 22212, The Republic of Korea

${ }^{d}$ School of Chemical and Biological Engineering, Seoul National University, Seoul 08826, The Republic of Korea

${ }^{e}$ Department of Chemical Engineering, Pohang University of Science and Technology (POSTECH), Pohang, Gyeongbuk 37673, The Republic of Korea

$\dagger$ Electronic supplementary information (ESI) available: Experimental details; Fig. S1-S20, displaying the quantum chemical calculation results, ${ }^{1} \mathrm{H}$ and ${ }^{13} \mathrm{C}$ $\left\{{ }^{1} \mathrm{H}\right\}$ NMR spectra, the ${ }^{1} \mathrm{H}$ NMR and the UV-vis absorption spectra obtained with increasing the concentration of PtN, the ECD spectra of the co-assemblies recorded upon rotating the sample, the ECD spectra for the co-assemblies containing increased concentrations of PtBox, steady-state photophysical and chiroptical behaviors of $(R)-/(S)$-PtBox, phosphorescence and photoluminescence excitation spectra of PtN, phosphorescence decay traces for the self-assemblies of PtN, phosphorescence spectra of the co-assemblies obtained with increasing the concentration of PtBox, temperature-dependent phosphorescence, UV-vis absorption and ECD spectra of the co-assemblies, and the circularly polarized phosphorescence spectra; Tables S1-S3, listing chiroluminescence data reported in literatures, photophysical data of PtN and $(R)-/(S)$-PtBox, and biexponential decay fit results of the traces shown in Fig. 5a. See DOI: 10.1039/c8sc04509g rotating either right-handedly or left-handedly. ${ }^{1}$ The circular enrichment is quantitated using a luminescence dissymmetry factor $g_{\text {lum }}=2\left(I_{\mathrm{LCPL}}-I_{\mathrm{RCPL}}\right) /\left(I_{\mathrm{LCPL}}+I_{\mathrm{RCPL}}\right)$, where $I_{\mathrm{LCPL}}$ and $I_{\mathrm{RCPL}}$ are the light intensities of the left- and right-handed CPL, respectively. Circularly polarized luminescence is valuable for its utility in elucidating various photofunctions. For example, 3D displays with a high luminance are possible using CP electroluminescence, ${ }^{2-4}$ because the two handedness directions can be used to create binocular disparities with minimal losses in the light intensity. ${ }^{5} \mathrm{CPL}$ is anticipated to find a broader range of photonic applications, including quantum encryption and security-enhanced optical communications. ${ }^{6,7}$

Exploiting the photonic utility of CPL requires zero-field emitters that produce high values of $\left|g_{\text {lum }}\right|$ and photoluminescence quantum yield (PLQY). Lanthanide complexes, such as $\left[\mathrm{Eu}\left(\mathrm{facam}_{3}\right)_{\text {(facam }}=3\right.$-(trifluoromethylhydroxymethylene)-(+)-camphorate), have been most successful, but their applicability is limited by inflexible syntheses and a lack of tunability. ${ }^{8}$ Two strategies have been pursued for the development of molecular CPL emitters. One strategy is based on asymmetric coupling between excitons within a molecular dyad; for example, CPL can be obtained through the asymmetric Davydov splitting of fluorophores, such as perylene diimide, ${ }^{9,10}$ 5,6-biscarbazolylphthalimide, ${ }^{11}$ terthiophene, ${ }^{12}$ pyrene, ${ }^{13}$ anthracene,${ }^{14}$ and azaacene,${ }^{15}$ in chiral arrangements. Exciton coupling is reinforced in aggregate states, leading to 
improvements in $\left|g_{\text {lum }}\right| \cdot{ }^{16}$ The other strategy used to develop molecular CPL emitters employs molecules bearing helical $\pi$ conjugation. Derivatives of helicene,,$^{3,17-27}$ 1,1'-binaphthalene ${ }^{28-31}$ paracyclophane,${ }^{32}$ chiral boron dipyrromethene, ${ }^{33,34}$ cyclic polyaromatic compounds, ${ }^{35}$ and chiral complexes of $\operatorname{Ir}(\mathrm{III})^{23,36-38}$ or Pt(II) $)^{39-43}$ have been found to display CPL activity. Although molecular factors that control the CPL behaviors of these molecules require further resolution, the helical structures may confer magnetic allowance to the electronic transition resulting in CPL emission.

Despite advances in the field, the development of molecular emitters of CPL has remained a significant challenge. The apparently necessary trade-off between $\left|g_{\text {lum }}\right|$ and PLQY presents a major obstacle to the full utilization of CPL photofunctions (see ESI, Table S1 $\uparrow$ for a list of $\left|g_{\text {lum }}\right|$ and PLQY values reported previously for CPL emitters). In addition, the two strategies tested thus far have required labor-intensive syntheses with poor flexibility for further elaboration. Therefore, there is a strong need for tractable strategies for obtaining CPL with high values of $\left|g_{\text {lum }}\right|$ and PLQY.

Herein, we report the generation and amplification of circularly polarized phosphorescence from helical assemblies of a simple, achiral molecule. Achiral monocycloplatinated complexes (soldiers) co-assemble into helical nanoribbons with a smaller fraction of chiral monocycloplatinated complexes (sergeants). The helical sense of the assembly is dictated by the sergeant (Fig. 1). The sergeants-and-soldiers co-assembly strategy improves $\left|g_{\text {lum }}\right|$ by two orders of magnitude and PLQY by one order of magnitude relative to the $\left|g_{\text {lum }}\right|$ of the chiral sergeant and the PLQY of the soldier, respectively. Our approach uniquely combines two previous strategies in that both asymmetric exciton coupling and the magnetic allowance in the electronic transition are facilitated in the co-assemblies.

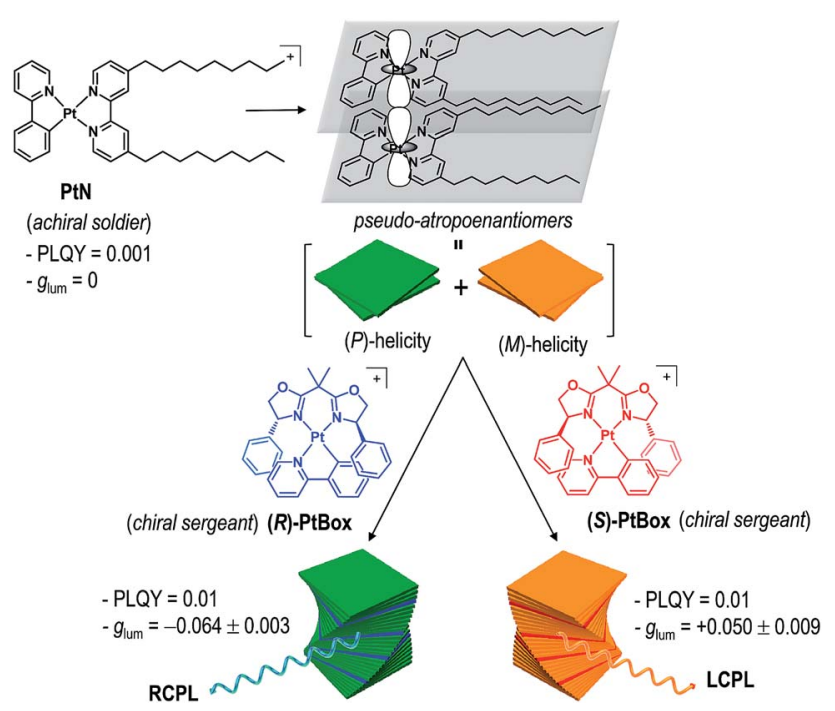

Fig. 1 Molecular strategy to obtaining circularly polarized phosphorescence from superhelical co-assemblies consisting of PtN soldiers and PtBox sergeants.

\section{Results and discussion}

\section{Chiroptical induction by controlling torsional displacement}

Our co-assembly strategy exploits the strong propensity of square planar $\mathrm{Pt}(\mathrm{II})$ complexes to form co-facial stacks. Pioneering studies by the groups of Che, Yam, De Cola, Kato, and Castellano established that the spontaneous assembly is driven by the synergetic effects of a Pt...Pt metallophilic interaction and a $\pi-\pi$ interaction among the cyclometalating ligands. ${ }^{\mathbf{4 4 - 5 2}}$ The resulting $\mathrm{Pt}(\mathrm{II})$ assemblies are useful in a variety of applications. ${ }^{53-57}$ We employed a Pt(II) complex having two nonyl chains, [Pt(2-phenylpyridinato) $\left(4,4^{\prime}\right.$-bis(nonyl)-2,2'-bipyridine)] $\mathrm{ClO}_{4}(\mathrm{PtN})$, as a soldier molecule (see Fig. 1 for the structure of $\mathrm{PtN}$ ). The nonyl chains provided torque that induced torsional displacement along the axis orthogonal to the molecular planes, thereby generating a helical PtN stack. The resulting PtN stacks were pseudo-atropo-enantiomeric because the formation of the right-handed (i.e., (P)-helical) and left-handed (i.e., $(M)$-helical) stacks was equally probable. Therefore, self-assemblies of PtN alone did not display any chiroptical activities (vide infra).

We anticipated that the addition of a chiral analogue of PtN (solider) would induce a Pfeiffer effect and enrich the enantiomeric excess in the helical PtN stacks. ${ }^{58}$ The initial PtN helical stack would serve as a homochiral seed for the hierarchical growth of superhelical assemblies because helical inversion is thermodynamically improbable. ${ }^{59}$ This strategy is advantageous over previous assembly approaches that employed co-assemblies of chiral gelators and an achiral fluorophore, ${ }^{60}$ as it improves both $\left|g_{\text {lum }}\right|$ and PLQY. In addition, the use of a simple achiral molecule as a CPL unit offers unique benefits over the selfassembly of homochiral CPL emitters..$^{10,16,17,19,40,41,60-70}$

As a first step in our study, we performed quantum chemical calculations of a dimeric stack of truncated PtN molecules at the CAM-B3LYP level of theory. The optimized PtN stack possessed the lowest energy metal-metal-to-ligand charge-transfer (MMLCT) singlet and triplet states, indicative of electronic coupling between the two PtN molecules (Fig. 2 and ESI, Fig. S1 $\dagger$ ). The MMLCT transition carried a non-negligible magnetic allowance. The electric $(\mu)$ and magnetic $(m)$ transition dipole moments of the MMLCT transition were parallel each other with an angle $(\theta)$ of $4.54^{\circ}$, yielding a rotatory strength $(R, R=|\mu| \cdot|m| \cos \theta)$ as large as $4.8 \times 10^{-39} \mathrm{erg}$ esu $\mathrm{cm}$ per Gauss (ESI, Fig. S2 $\dagger$ ). The parallel disposition between $\mu$ and $m$ markedly contrasts with the cases of single molecular CPL emitters. As expected, the $R$ of the MMLCT transition of the PtN stack oscillated between positive and negative values as the top $\mathrm{PtN}$ molecule rotated relative to the bottom PtN molecule while maintaining a fixed Pt-Pt distance of $3.7 \AA$ (Fig. 2). Although this calculation was limited to a dimer, ${ }^{71}$ it supported our claim that the chiroptical activities could be controlled by dihedral rotation within the $\mathrm{PtN}$ assemblies.

\section{Formation of superhelical co-assemblies by Pfeiffer effect}

To enable our study, we synthesized an enantiomeric pair of sergeant Pt(II) complexes having chiral bisoxazoline ligands in place of 4,4'-bis(nonyl)-2,2'-bipyridine, [Pt(2-phenylpyridinato) $((+)-$ 


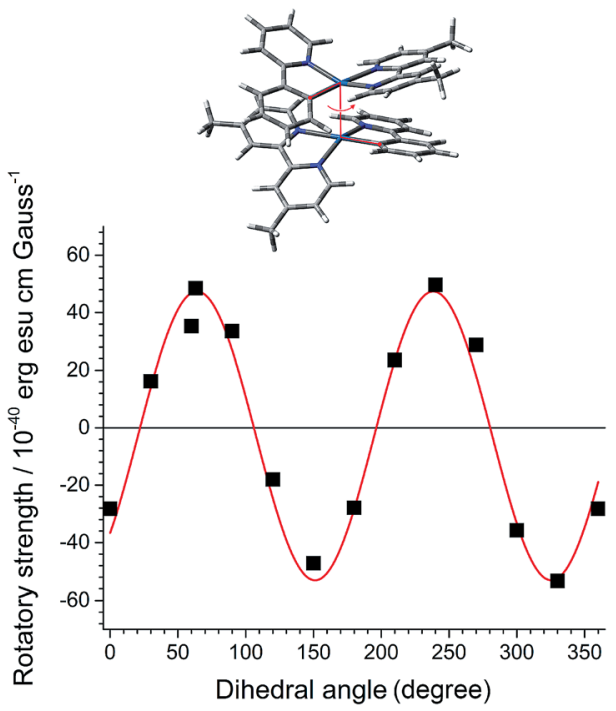

Fig. 2 Torsional control of chiroptical properties. Simulation of the rotatory strength $(R)$ of the MMLCT transition as a function of the dihedral angle of a dimer comprising a truncated form of the PtN molecules (top structure). The red oscillatory curve provides a visual guide. Calculation conditions: TD-CAM-B3LYP/LANL2DZ:6$311+G(d, p) / / C A M-B 3 L Y P / L A N L 2 D Z: 6-311+G(d, p)$. Twenty states were considered for the TD-DFT calculations.

2,2-isopropylidenebis((4R)-4-phenyl-2-oxazoline) $)] \mathrm{ClO}_{4} \quad((R)$-PtBox $)$ and $\quad[\mathrm{Pt}(2-$ phenylpyridinato $)((-)-2,2-$ isopropylidenebis $((4 S)-4-$ phenyl-2-oxazoline))] $\mathrm{ClO}_{4}$ ((S)-PtBox). These complexes were prepared from a dinuclear cycloplatinated precursor, following the standard chloride abstraction method that employed $\mathrm{AgClO}_{4}$ (Scheme 1). The synthetic procedures are described in the ESI. $\dagger$ Structural identification data, obtained using ${ }^{1} \mathrm{H}$ and ${ }^{13} \mathrm{C}\left\{{ }^{1} \mathrm{H}\right\}$ NMR spectroscopy, high-resolution mass spectrometry, and elemental analyses, were fully consistent with the proposed structures (ESI, Fig. S3-S8†).
Co-assemblies were grown on quartz substrates by slow evaporation of a 1,2-dichloroethane solution containing PtBox and PtN (2.0 wt $\%$ total solute; PtBox : PtN $=2: 5, \mathrm{w} / \mathrm{w}$ ) under atmospheric pressure at room temperature. As shown in Fig. 3a, confocal laser-scanning micrographs $\left(\lambda_{\mathrm{ex}}=405 \mathrm{~nm}, \lambda_{\mathrm{obs}}=470-\right.$ $700 \mathrm{~nm}$ ) revealed the formation of homohelical nanoribbons of the co-assemblies. The helical sense was determined exclusively by the identity of the added PtBox; $(R)$-PtBox and $(S)$-PtBox furnished $(P)$-helical and $(M)$-helical nanoribbons, respectively. By contrast, PtN self-assemblies did not contain any helical structures. Helical control over the co-assemblies was further supported by fieldemission scanning electron microscopy (FE-SEM) images (Fig. 3b).

Note that the widths of the nanoribbons $(2-5 \mu \mathrm{m})$ were much greater than the calculated molecular dimensions of PtN $(20.8 \AA$ $\times 9.0 \AA$ ). . The AFM topographies indicated the formation of assemblies with a step height of $2.2 \mathrm{~nm}$ (Fig. 3c). Obviously, this height was longer than the longest dimension of PtN (i.e., 20.8 ), which suggested the interdigitation of PtN molecules within the assemblies. The packing structures were elucidated using 2D grazing-incident X-ray diffraction (2D GIXD) studies. As shown in Fig. 3d, 2D GIXD patterns of the PtN and PtBox/PtN assemblies produced highly oriented X-ray reflections, corresponding to the (h00) reflections along the $Q_{z}$ axis and Braggs peaks vertically along the $Q_{z}$ direction at a given $Q_{x y}$. These reflection patterns were indicative of pseudo hexagonal close packing of PtN cylinders. Analyses of the 2D GIXD patterns revealed a monoclinic lattice structure $\left(a=24.66 \AA, b=27.09 \AA, \gamma=117^{\circ}\right)$, where the PtN cylinders were oriented parallel to the surface (see the bottom structures in Fig. 3). On the contrary, an orthorhombic structure developed in the PtN self-assemblies. The X-ray reflection of (001) at $Q_{x y}=1.708 \AA^{-1}(d=3.680 \AA)$ corresponded to the intermolecular distance between PtN molecules within the cylinder. This distance is slightly longer than the lengths of typical $\mathrm{Pt} \cdots \mathrm{Pt}$ metallophilic interactions $(<3.5 \AA),{ }^{49}$ indicating weak $\mathrm{d}_{z^{2}}-\mathrm{d}_{z^{2}}$ orbital overlap. However, the distance was consistent with the redshifted MMLCT emission (vide infra).

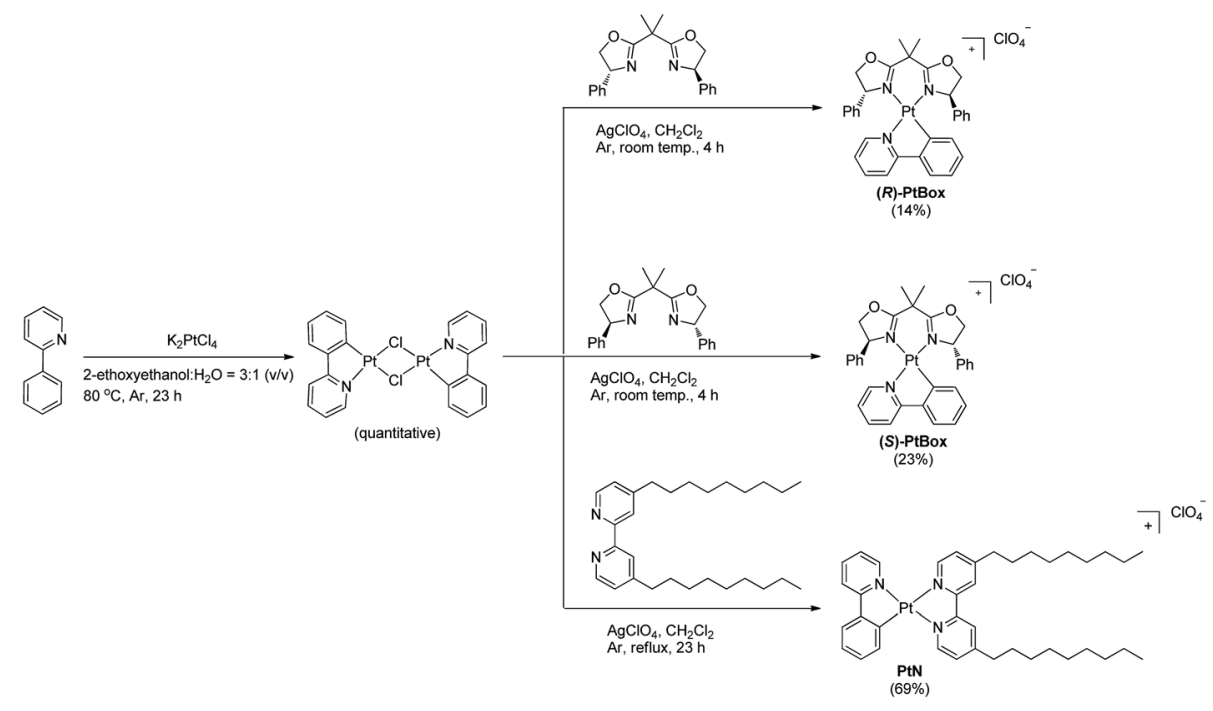

Scheme 1 Syntheses of (R)-PtBox, (S)-PtBox and PtN 
(R)-PtBox/PtN
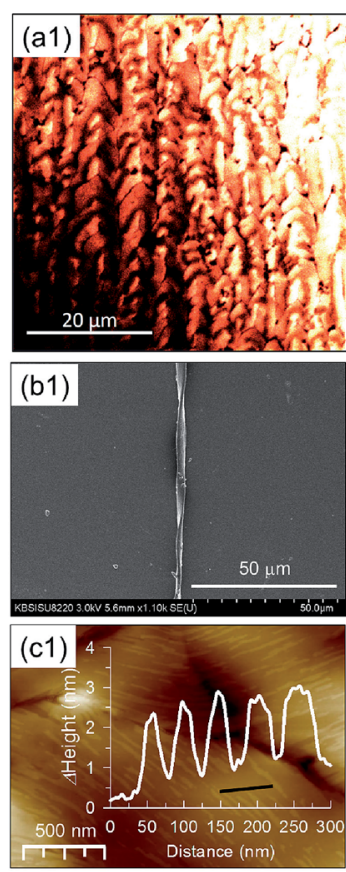

$Q_{\mathrm{z}}$
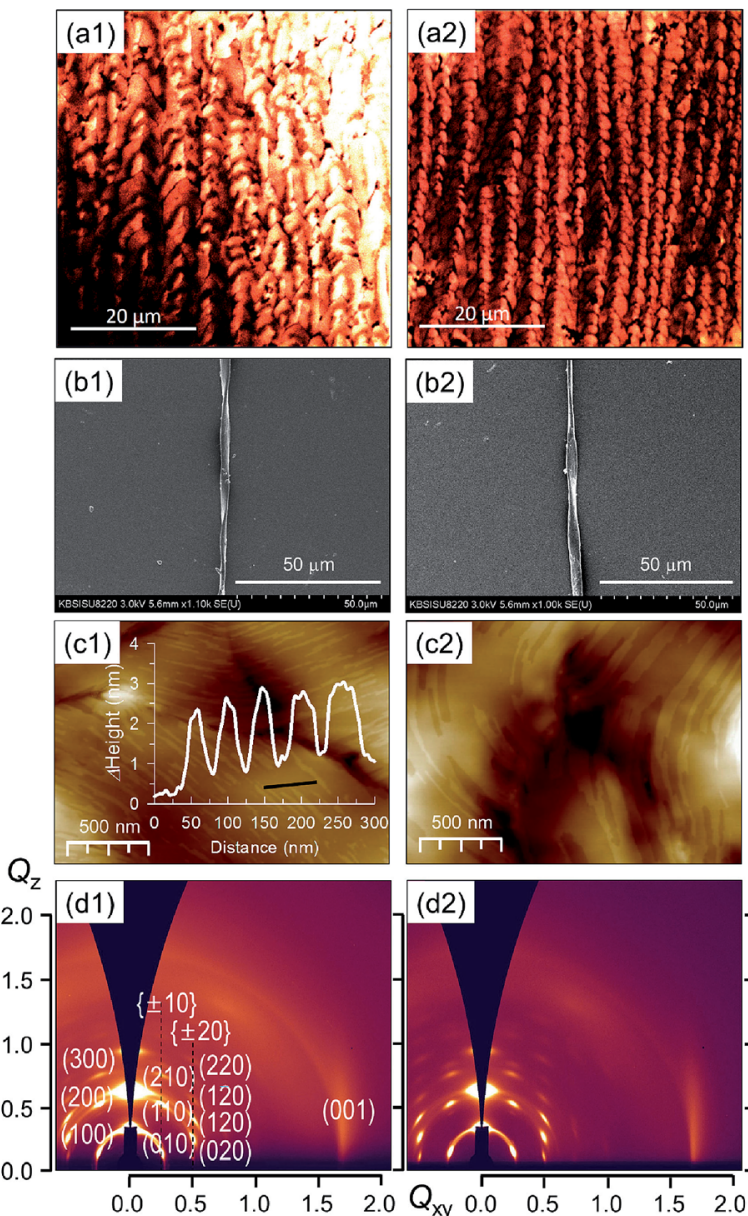

\section{(b3)}
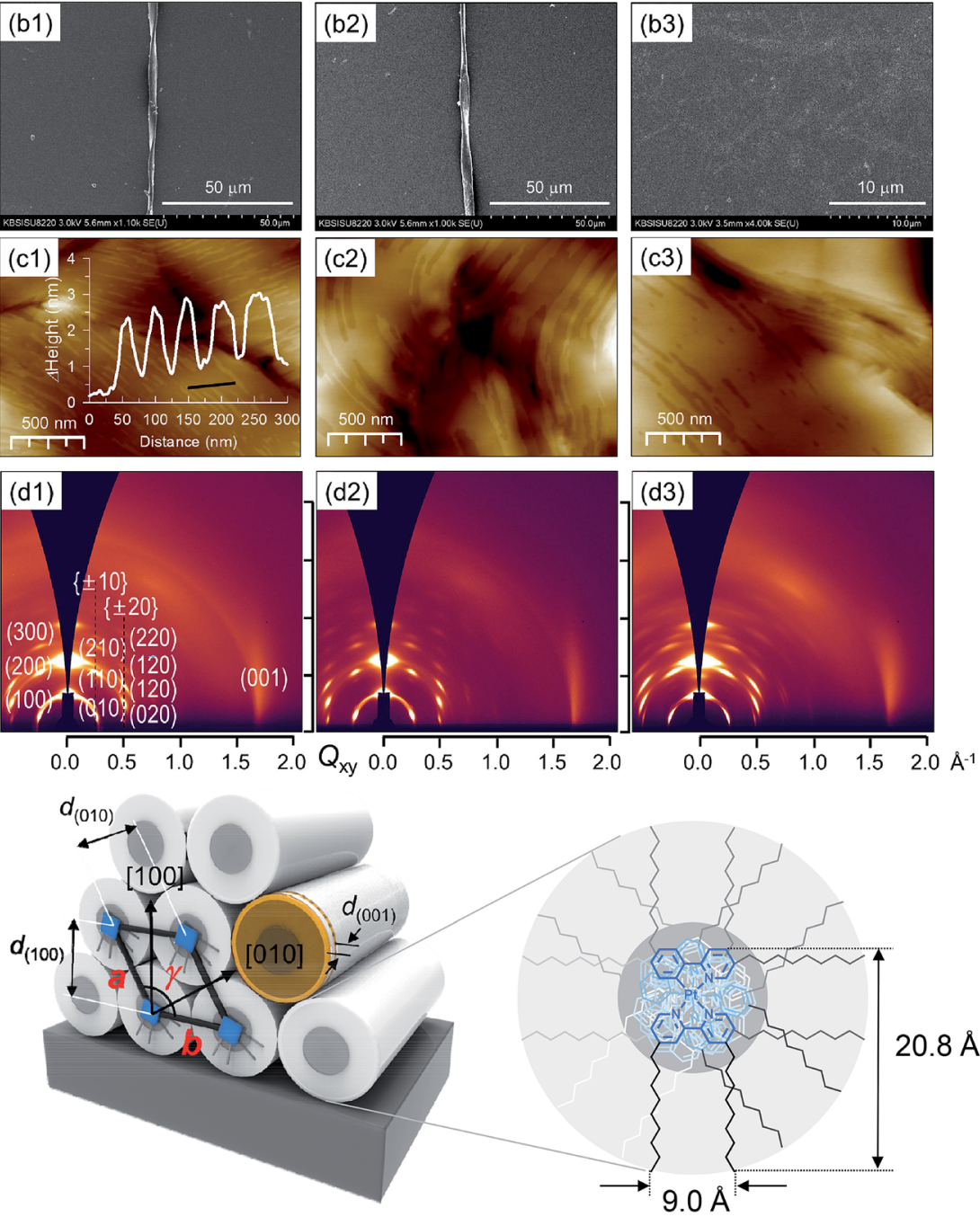

$20.8 \AA$

Fig. 3 Chiral amplification in the co-assemblies of PtBox/PtN. The co-assemblies were grown by drop-casting a 1,2-dichloroethane solution (2 wt\% total solute) containing PtN and (R)- (a1, b1, c1, and d1) or (S)-PtBox (a2, b2, c2, and d2) (PtBox : PtN = $2: 5$, w/w). Self-assemblies of PtN were grown identically (a3, b3, c3, and d3). (a) Confocal laser-scanning micrographs of the PtN assemblies prepared in the presence of $(R)$-PtBox (a1) or (S)-PtBox (a2) and in the absence of PtBox (a3). $\lambda_{\mathrm{ex}}=405 \mathrm{~nm} . \lambda_{\mathrm{em}}=470-700 \mathrm{~nm}$. (b) FE-SEM images and (c) AFM topographies of the coassemblies prepared in the presence of $(R)$-PtBox $(b 1, c 1)$ or $(S)$-PtBox $(b 2, c 2)$ and in the absence of PtBox (b3, c3). The inset graph depicts the step profile along the black line. (d) 2D GIXD patterns obtained from assemblies of PtN prepared in the presence of $(R)$ - PtBox (d1) or (S)-PtBox (d2) and in the absence of PtBox (d3). The bottom structures show a monoclinic structure of the PtBox/PtN assemblies established from the 2D GIXD results. The lattice parameters, $a, b$ and $\gamma$, are marked in red.

\section{Amplification of chiroptical properties}

The co-assemblies included Pt $\cdots$ Pt metallophilic interactions, as was evident by the emergence of the weak MMLCT transition at a wavelength of $500 \mathrm{~nm}$ in the UV-vis absorption spectra (Fig. 4a). ${ }^{1} \mathrm{H}$ NMR experiments corroborated this notion (see the results and discussion in ESI, Fig. S9†). The association constant $\left(K_{\mathrm{a}}\right)$ for the PtN assembly was estimated to be $<100$ $\mathrm{M}^{-1}$, based on the measured concentration-dependent increase in the MMLCT absorbance (ESI, Fig. S10 $†$ ). Although the MMLCT transition made non-negligible contribution, as shown 
(a)

(b)

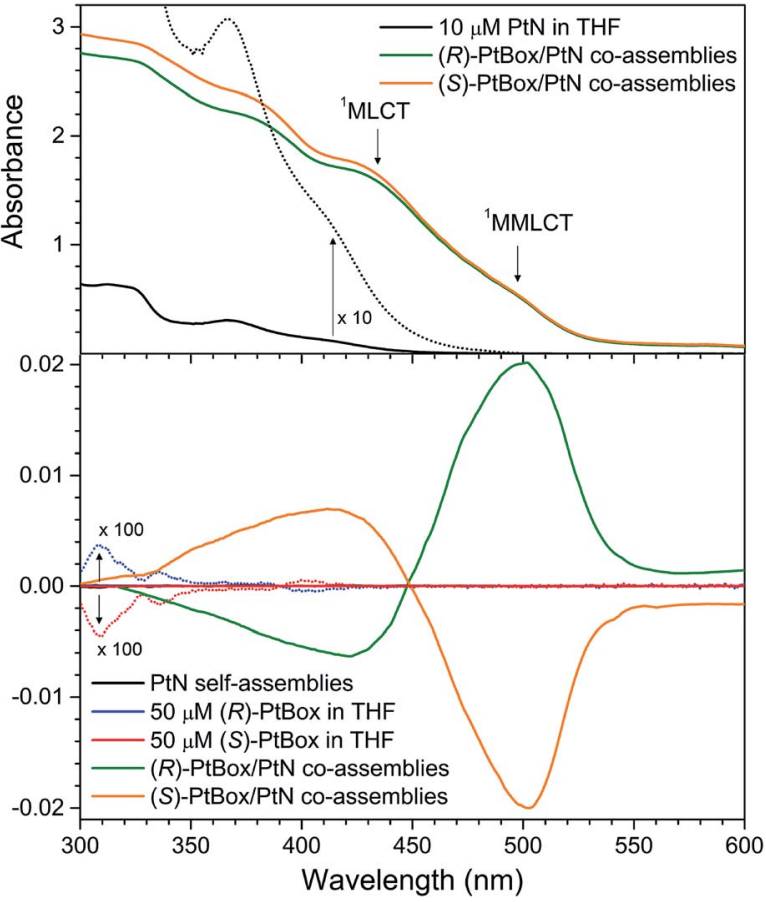

Fig. 4 Induction and amplification of electronic circular dichroism (ECD). (a) UV-vis absorption spectra of $10 \mu \mathrm{M}$ PtN (THF, black) and coassemblies of $(R)$-PtBox/PtN (2:5, w/w; green), and (S)-PtBox/PtN (2: 5, w/w; orange). (b) ECD spectra obtained from self-assembled PtN (black), $50 \mu \mathrm{M}(R)$-PtBox (THF, blue), $50 \mu \mathrm{M}(S)$-PtBox (THF, red), or co-assemblies of (R)-PtBox/PtN (green) and (S)-PtBox/PtN (orange). Absorption dissymmetry factor $\left(g_{\text {abs }}\right)$ : solution and self-assembly of PtN, 0; $(R)$-PtBox, $+3.7 \times 10^{-5}(308 \mathrm{~nm}) ;(S)$-PtBox, $-4.3 \times 10^{-5}(308$ $\mathrm{nm})$; co-assembly of $(R)$-PtBox/PtN, $+0.020(500 \mathrm{~nm})$; co-assembly of (S)-PtBox/PtN, -0.020 (500 nm).

in Fig. 4b, the electronic circular dichroism (ECD) spectra of the co-assemblies were dominated by the strong bisignate signals of the metal-to-ligand charge-transfer (MLCT) band of PtN at a wavelength of $450 \mathrm{~nm}$. The chiral exciton theory dictates that $(P)$-helical co-assemblies displayed a positive Cotton effect, whereas $(M)$-helical co-assemblies displayed a negative Cotton effect. ${ }^{1}$ Therefore, judging from the bisignate signals in Fig. $4 \mathrm{~b}$, it was concluded that $(R)-\mathrm{PtBox} / \mathrm{PtN}$ and $(S)-\mathrm{PtBox} / \mathrm{PtN}$ coassemblies were in $(P)$ - and $(M)$-helical sense, respectively. Note that the MLCT transition was unimolecular, whereas the MMLCT transition was supramolecular. These chiroptical behaviors, thus, suggested that the asymmetric coupling among MLCT excitons dominated the magnetically allowed MMLCT transition in the chiral photon absorption process. The ECD signals were devoid of linear dichroism or birefringence because the ECD spectra were invariant under the rotation of the azimuthal angle of the sample (ESI, Fig. S11 $\dagger$ ).

The Kuhn dissymmetry factor $g_{\text {abs }}$, defined as $g_{\text {abs }}=2\left(\varepsilon_{\mathrm{L}}-\varepsilon_{\mathrm{R}}\right) /$ $\left(\varepsilon_{\mathrm{L}}+\varepsilon_{\mathrm{R}}\right)\left(\varepsilon_{\mathrm{L}}\right.$ and $\varepsilon_{\mathrm{R}}$ are the ellipticity of the left- and right-handed circularly polarized absorption), in the ECD spectra increased in proportion to the amount of PtBox added to form the coassemblies, and it reached a plateau at PtBox mole fraction $([\mathrm{PtBox}] /([\mathrm{PtBox}]+[\mathrm{PtN}]))$ of 0.2 (ESI, Fig. S12†). This result indicated chiral amplification based on at least four PtN solider molecules following one PtBox sergeant during the growth of the homohelical co-assemblies. The largest $g_{\text {abs }}$ values were found to be +0.020 and -0.020 at $500 \mathrm{~nm}$ for co-assemblies of $(R)$-PtBox/ PtN and (S)-PtBox/PtN (PtBox: PtN $=2: 5, \mathrm{w} / \mathrm{w}$ ), respectively. These values were three orders of magnitude greater than the largest $g_{\text {abs }}$ for $(R)$-PtBox $\left(g_{\text {abs }}=+3.7 \times 10^{-5}\right)$ and $(S)$-PtBox $\left(g_{\text {abs }}=\right.$ $-4.3 \times 10^{-5}$ ), indicating the effectiveness of the asymmetric exciton coupling of PtN molecules in the co-assemblies (ESI, Fig. S13 and Table S2†). As expected, self-assemblies of PtN (i.e., no PtBox) did not produce any ECD signals (Fig. $4 \mathrm{~b}$ ).

The PtBox/PtN co-assemblies produced a broad phosphorescence spectrum with an emission peak wavelength $\left(\lambda_{\mathrm{em}}\right)$ of $615 \mathrm{~nm}$ and a phosphorescence lifetime $\left(\tau_{\mathrm{obs}}\right)$ of $0.24 \pm 0.05 \mu \mathrm{s}$ (Table 1). The phosphorescence emission resulted from the triplet MMLCT transition in the PtN assemblies, which was identical to the emission observed in PtN self-assemblies, and differed from the phosphorescence emission observed from a dilute solution of $\mathrm{PtN}\left(\lambda_{\mathrm{em}}=480 \mathrm{~nm} ; 10 \mu \mathrm{M}\right.$ in 1,2-dichloroethane) (ESI, Fig. S14†). The photoluminescence excitation (PLE) spectrum corresponding to the $615 \mathrm{~nm}$ phosphorescence emission overlapped with the MMLCT absorption band, supporting this notion (ESI, Fig. S15†). In addition, the transient photoluminescence traces were devoid of a delayed rise-anddecay profile, excluding the possibility that an excimer was responsible for the $615 \mathrm{~nm}$ phosphorescence emission (ESI, Fig. S16†) ${ }^{72}$ Neither $(R)$-PtBox nor $(S)$-PtBox molecules displayed chromic shifts upon self-aggregate formation, eliminating the possibility that excitonic interactions among sergeants contributed to the $615 \mathrm{~nm}$ phosphorescence emission.

The enhancement in the PLQY was notable. The absolute PLQY values obtained from both $(R)$-PtBox/PtN and $(S)$-PtBox/ PtN co-assemblies under air-equilibrated conditions were 0.01, one order of magnitude greater than the value $(<0.001)$ recorded in a deaerated solution of PtN. This increase resulted from the acceleration of the radiative process by the MMLCT transition. Indeed, the radiative rate $\left(k_{\mathrm{r}}\right)$ of the MMLCT phosphorescence of the co-assemblies $\left(\lambda_{\mathrm{em}}=615 \mathrm{~nm}\right)$ was $9.8 \times 10^{3} \mathrm{~s}^{-1}$, whereas a one order of magnitude slower $k_{\mathrm{r}}\left(0.83 \times 10^{3} \mathrm{~s}^{-1}\right)$ was obtained for the MLCT phosphorescence of a PtN molecule. The non-radiative rate $\left(k_{\mathrm{nr}}\right)$ values of the MMLCT and MLCT transitions did not vary significantly $\left(k_{\mathrm{nr}}=1.62 \times 10^{6} \mathrm{~s}^{-1}\right.$ (MMLCT) vs. $0.83 \times 10^{6} \mathrm{~s}^{-1}$ (MLCT)), indicating radiative control.

In addition to the radiatively boosted PLQY, ultrafast energy transfer from PtBox to PtN contributed to the phosphorescence emission of the co-assemblies. As shown in Fig. 5a, $(R)$-PtBox and (S)-PtBox exhibited long-lived luminescence with $\tau_{\text {obs }}$ values of 3.4 $\mu \mathrm{s}$ and $3.7 \mu \mathrm{s}$, respectively. Incorporating PtBox into the coassemblies significantly shortened $\tau_{\text {obs }}$ by enabling energy transfer to PtN: $\tau_{\text {obs }}$ decreased to $0.011 \mu$ s in both $(R)$ - and $(S)$-PtBox/ PtN. The energy-transfer rates $\left(k_{\mathrm{ET}} \mathrm{S}\right)$ were estimated to be $9.1 \times$ $10^{7} \mathrm{~s}^{-1}$ for both (R)-PtBox and (S)-PtBox, approximately four and two orders magnitude greater than $k_{\mathrm{r}}\left(\sim 10^{3} \mathrm{~s}^{-1}\right)$ and $k_{\mathrm{nr}}\left(\sim 10^{5}\right.$ to $10^{6} \mathrm{~s}^{-1}$ ), respectively (see ESI, Table S2 $\uparrow$ for the $k_{\mathrm{r}}$ and $k_{\mathrm{nr}}$ values). The ultrafast intra-assembly energy transfer was further supported by the observation of increased phosphorescence intensities from co-assemblies containing increased concentrations of PtBox (ESI, Fig. S17†). 
Table 1 Photophysical data for the assemblies of PtN, (R)-PtBox/PtN and (S)-PtBox/PtN

\begin{tabular}{|c|c|c|c|c|c|c|c|c|}
\hline & $\lambda_{\mathrm{abs}}{ }^{a}(\mathrm{~nm})$ & $\lambda_{\mathrm{em}}^{b}(\mathrm{~nm})$ & $\mathrm{PLQY}^{c}$ & $\tau_{\text {avg }}{ }^{d}(\mu \mathrm{s})$ & $k_{\mathrm{r}}^{e}\left(10^{4} \mathrm{~s}^{-1}\right)$ & $k_{\mathrm{nr}}^{f}\left(10^{6} \mathrm{~s}^{-1}\right)$ & $g_{\text {abs }}{ }^{g}$ & $g_{\text {lum }}{ }^{h}$ \\
\hline PtN self-assemblies & 490 & 615 & 0.006 & 0.61 & 0.98 & 1.62 & N.A. & N.A. \\
\hline (R)-PtBox/PtN co-assemblies & 490 & 615 & 0.01 & 0.24 & 4.2 & 4.1 & +0.02 & $-0.064 \pm 0.003$ \\
\hline
\end{tabular}

(S)-PtBox/PtN co-assemblies

${ }^{a}$ Absorption peak wavelength. ${ }^{b}$ Photoluminescence peak wavelength. Excitation wavelengths $=370 \mathrm{~nm}(\mathrm{PtN})$ and $326 \mathrm{~nm}((R)$ - and $(S)$-PtBox). ${ }^{c}$ Absolute photoluminescence quantum yields determined with employing an integrating sphere. ${ }^{d}$ Photoluminescence lifetime obtained after pulsed laser excitation at $377 \mathrm{~nm}$ (temporal resolution $=1.6 \mathrm{~ns}$; observation wavelength $\left.\left(\lambda_{\text {obs }}\right)=615 \mathrm{~nm}\right)($ PtN self-assemblies) and $615 \mathrm{~nm}$ (PtBox/PtN co-assemblies). A biexponential decay model was used: photoluminescence intensity $=A_{1} \exp \left(-t / \tau_{1}\right)+A_{2} \exp \left(-t / \tau_{2}\right)+A_{0} \cdot \tau_{\mathrm{avg}}=$ $\left(\sum A_{\mathrm{i}} \tau_{\mathrm{i}}{ }^{2}\right) /\left(\sum A_{\mathrm{i}} \tau_{\mathrm{i}}\right)(i=1-2) .{ }^{e}$ Radiative rate constant, $k_{\mathrm{r}}=\mathrm{PLQY} / \tau_{\mathrm{avg}} .{ }^{f}$ Non-radiative rate constant, $k_{\mathrm{nr}}=(1-\mathrm{PLQY}) / \tau_{\mathrm{avg}} .{ }^{g}$ Absorption dissymmetry factor. $\lambda_{\text {obs }}=500 \mathrm{~nm} .{ }^{h}$ Luminescence dissymmetry factor. $\lambda_{\text {obs }}=576 \mathrm{~nm}$.

(a)

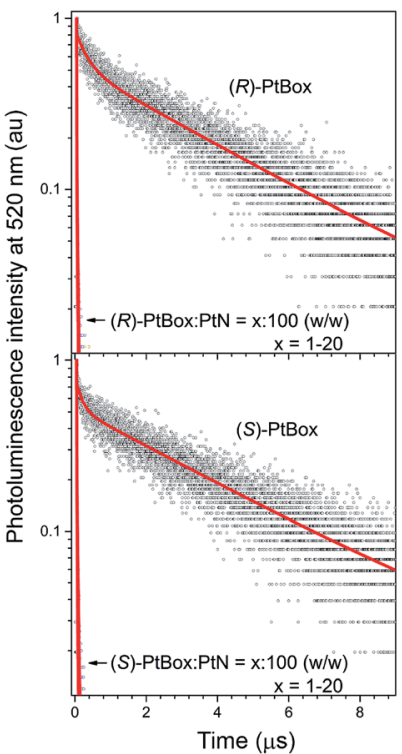

(b)

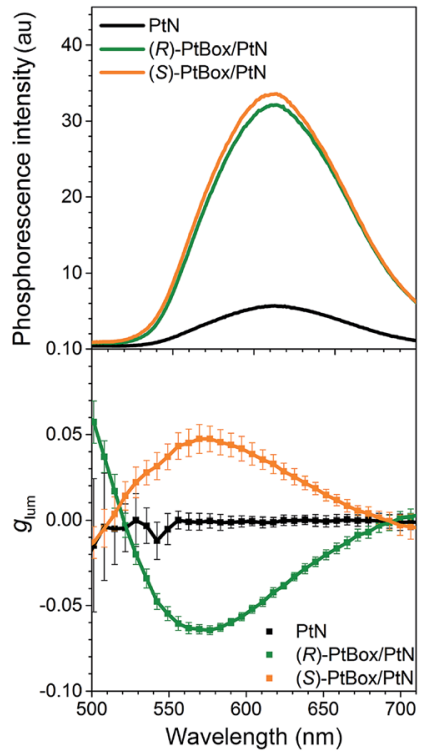

Fig. 5 Amplified circularly polarized phosphorescence. (a) Comparisons of the photoluminescence decay traces of $(R)$ - or (S)-PtBox in Arsaturated THF $(50 \mu \mathrm{M})$ or of the co-assemblies (PtBox : PtN $=x: 100$, $\mathrm{W} / \mathrm{w} ; x=1-20)$, observed at $520 \mathrm{~nm}$ after pulsed laser photoexcitation at $377 \mathrm{~nm}$ (temporal resolution =1.6 ns). Top, $(R)$-PtBox; bottom, $(S)$ PtBox. The red curves show fits to a biexponential decay model. The fit results are summarized in ESI, Table S3. $\dagger$ (b) Steady-state phosphorescence emission (top, $\lambda_{\mathrm{ex}}=363 \mathrm{~nm}$ ) and plots of $g_{\text {lum }}$ values as a function of the emission wavelength (bottom) for the self-assemblies of PtN (black) and the co-assemblies of (R)-PtBox/PtN (green) or (S)PtBox/PtN (orange). The CPL measurements were repeated 50 times for each fresh sample, and the average and standard deviations are shown.

The MMLCT phosphorescence disappeared upon heating. An abrupt decrease in the MMLCT phosphorescence emission was observed at $343 \mathrm{~K}$, suggesting the presence of cooperative assemblies of PtN molecules (ESI, Fig. S18†). ${ }^{73}$ The endothermic phosphorescence decrease accompanied losses in the bisignate Cotton effect in the ECD spectra and in the MMLCT absorption in the UV-vis absorption spectra of the $(R)$-PtBox/ PtN co-assemblies (ESI, Fig. S19†). These results suggested thermally activated disassembly. The disassembly temperature could be shifted by varying the length of the alkyl chains in PtN.

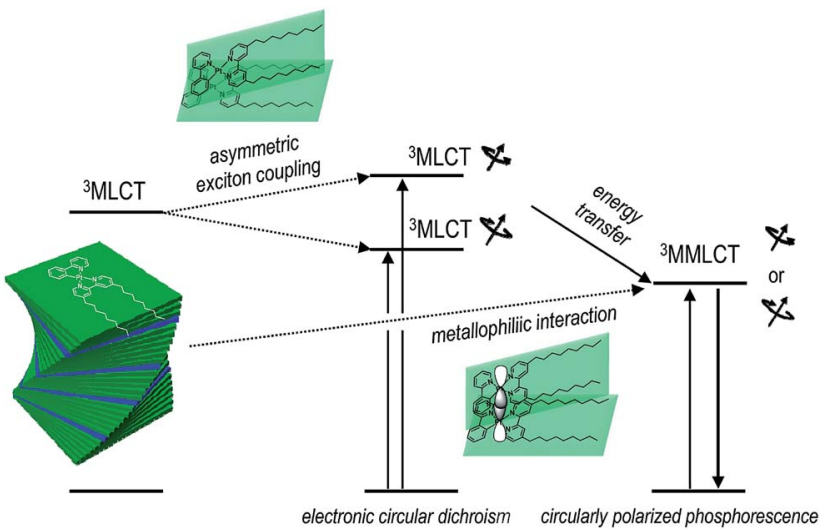

Fig. 6 Plausible mechanism underlying the photophysical processes that produce circularly polarized phosphorescence in the coassemblies.

As a final phase of our study, circularly polarized phosphorescence was recorded from the co-assemblies over the emission range 500-710 nm using a linear polarizer and a rotating quarter-wave retarder. The validity of our detection method was examined using $\left[\mathrm{Eu}(\text { facam })_{3}\right]$ as a standard. Perfect sinusoidal oscillations and identical phosphorescence intensities were obtained from $(R)$-PtBox/PtN and $(S)$-PtBox/PtN assemblies when the axis between the polarizer and the retarder was rotated (ESI, Fig. S20 $\dagger$ ), suggesting that linearly polarized emission contributed minimally to the CPL. As shown in Fig. 5b, the circularly polarized phosphorescence dissymmetry spectra overlapped with the arbitrary phosphorescence spectra obtained without a polarizer and a retarder. The coincidence indicated that the triplet MMLCT state was responsible for the

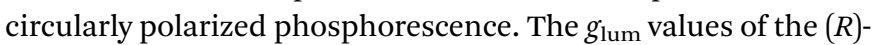
PtBox/PtN and $(S)$-PtBox/PtN co-assemblies were $-0.064 \pm 0.003$ and $+0.050 \pm 0.009$, respectively. These values were at least one order of magnitude greater than those obtained from $(R)$-PtBox $\left(\left|g_{\text {lum }}\right|<0.001\right)$ and $(S)$-PtBox $\left(\left|g_{\text {lum }}\right|<0.001\right)$.

\section{Conclusions}

We devised and demonstrated the molecular strategy to overcoming the trade-off problem in circularly polarized luminescence. The formation of hierarchical helix assemblies of 
cycloplatinated complexes improved both the luminescence dissymmetry factor and the quantum yield. The sergeants-andsoldiers principle was employed in the creation of the superhelical assemblies of cycloplatinated complexes. The principle is reminiscent of that employed in chiral nematic liquid crystals; ${ }^{74}$ however, the strong Pfeiffer effect associated with the generation and amplification of circularly polarized phosphorescence is unprecedented. The chiroptical amplification was ascribed to both the magnetically allowed MMLCT state for phosphorescence emission and enantiomeric enrichment of the circular polarization via absorption through the asymmetrically coupled MLCT states prior to populating the emitting MMLCT state (vide supra). Therefore, our co-assemblies were characterized by a unique three level system: Absorption of circularly polarized photons occurred through a transition to the asymmetrically exciton-coupled MLCT state, whereas circularly polarized phosphorescence resulted from the magnetically allowed MMLCT transition (see Fig. 6). Note that $g_{\text {lum }}$ and $g_{\text {abs }}$ values for the co-assemblies had opposite signs. The reversal in the dissymmetry factors may support our claim that the MLCT and MMLCT transitions associated with absorption and emission of CPL, respectively, functioned independently of one another. It is envisioned that decoupling the chiroptical absorption and emission processes could expand our ability to control circularly polarized light.

\section{Conflicts of interest}

There are no conflicts to declare.

\section{Acknowledgements}

This work was supported by a grant from the Samsung Research Funding Center for Future Technology (SRFC-MA1602-03). H. Y. acknowledges the grant provided by the National Research Foundation of Korea (NRF2017R1A2B4009313).

\section{Notes and references}

1 J. P. Riehl and F. S. Richardson, Chem. Rev., 1986, 86, 1-16.

2 F. Zinna, U. Giovanella and L. Di Bari, Adv. Mater., 2015, 27, 1791-1795.

3 J. R. Brandt, X. Wang, Y. Yang, A. J. Campbell and M. J. Fuchter, J. Am. Chem. Soc., 2016, 138, 9743-9746.

4 E. Peeters, M. P. T. Christiaans, R. A. J. Janssen, H. F. M. Schoo, H. P. J. M. Dekkers and E. W. Meijer, J. Am. Chem. Soc., 1997, 119, 9909-9910.

5 T. Kim, S. Kim, M.-J. Gim and S.-W. Choi, J. Korean Phys. Soc., 2013, 62, 713-717.

6 C. Wagenknecht, C.-M. Li, A. Reingruber, X.-H. Bao, A. Goebel, Y.-A. Chen, Q. Zhang, K. Chen and J.-W. Pan, Nat. Photonics, 2010, 4, 549-552.

7 J. F. Sherson, H. Krauter, R. K. Olsson, B. Julsgaard, K. Hammerer, I. Cirac and E. S. Polzik, Nature, 2006, 443, 557-560.

8 R. Carr, N. H. Evans and D. Parker, Chem. Soc. Rev., 2012, 41, 7673-7686.
9 H. Tsumatori, T. Nakashima and T. Kawai, Org. Lett., 2010, 12, 2362-2365.

$10 \mathrm{~J}$. Kumar, H. Tsumatori, J. Yuasa, T. Kawai and T. Nakashima, Angew. Chem., Int. Ed., 2015, 54, 5943-5947.

11 M. Li, S.-H. Li, D. Zhang, M. Cai, L. Duan, M.-K. Fung and C.-F. Chen, Angew. Chem., Int. Ed., 2018, 57, 2889-2893.

12 B. M. W. Langeveld-Voss, D. Beljonne, Z. Shuai, R. A. J. Janssen, S. C. J. Meskers, E. W. Meijer and J.-L. Bredas, Adv. Mater., 1998, 10, 1343-1348.

13 Y. Mimura, T. Nishikawa, R. Fuchino, S. Nakai, N. Tajima, M. Kitamatsu, M. Fujiku and Y. Imai, Org. Biomol. Chem., 2017, 15, 4548-4553.

14 J. Han, P. Duan, X. Li and M. Liu, J. Am. Chem. Soc., 2017, 139, 9783-9786.

15 Y. Inoue, D. Sakamaki, Y. Tsutsui, M. Gon, Y. Chujo and S. Seki, J. Am. Chem. Soc., 2018, 140, 7152-7158.

16 J. Kumar, T. Nakashima and T. Kawai, J. Phys. Chem. Lett., 2015, 6, 3445-3452.

17 K. E. S. Phillips, T. J. Katz, S. Jockusch, A. J. Lovinger and N. J. Turro, J. Am. Chem. Soc., 2001, 123, 11899-11907.

18 J. E. Field, G. Muller, J. P. Riehl and D. Venkataraman, J. Am. Chem. Soc., 2003, 125, 11808-11809.

19 T. Kaseyama, S. Furumi, X. Zhang, K. Tanaka and M. Takeuchi, Angew. Chem., Int. Ed., 2011, 50, 3684-3687.

20 K. Nakamura, S. Furumi, M. Takeuchi, T. Shibuya and K. Tanaka, J. Am. Chem. Soc., 2014, 136, 5555-5558.

21 H. Sakai, S. Shinto, J. Kumar, Y. Araki, T. Sakanoue, T. Takenobu, T. Wada, T. Kawai and T. Hasobe, J. Phys. Chem. C, 2015, 119, 13937-13947.

22 D.-Q. He, H.-Y. Lu, M. Li and C.-F. Chen, Chem. Commun., 2017, 53, 6093-6096.

23 N. Hellou, M. Srebro-Hooper, L. Favereau, F. Zinna, E. Caytan, L. Toupet, V. Dorcet, M. Jean, N. Vanthuyne, J. A. G. Williams, L. Di Bari, J. Autschbach and J. Crassous, Angew. Chem., Int. Ed., 2017, 56, 8236-8239.

24 T. Otani, A. Tsuyuki, T. Iwachi, S. Someya, K. Tateno, H. Kawai, T. Saito, K. S. Kanyiva and T. Shibata, Angew. Chem., Int. Ed., 2017, 56, 3906-3910.

25 M. Li, H.-Y. Lu, C. Zhang, L. Shi, Z. Tang and C.-F. Chen, Chem. Commun., 2016, 52, 9921-9924.

26 L. Fang, M. Li, W.-B. Lin, Y. Shen and C.-F. Chen, J. Org. Chem., 2017, 82, 7402-7409.

27 M. Li, C. Zhang, L. Fang, L. Shi, Z. Tang, H.-Y. Lu and C.-F. Chen, ACS Appl. Mater. Interfaces, 2018, 10, 8225-8230.

28 S. Feuillastre, M. Pauton, L. Gao, A. Desmarchelier, A. J. Riives, D. Prim, D. Tondelier, B. Geffroy, G. Muller, G. Clavier and G. Pieters, J. Am. Chem. Soc., 2016, 138, 3990-3993.

29 R. Aoki, R. Toyoda, J. F. Kogel, R. Sakamoto, J. Kumar, Y. Kitagawa, K. Harano, T. Kawai and H. Nishihara, J. Am. Chem. Soc., 2017, 139, 16024-16027.

30 J. Han, S. Guo, J. Wang, L. Wei, Y. Zhuang, S. Liu, Q. Zhao, X. Zhang and W. Huang, Adv. Opt. Mater., 2017, 5, 1700359.

31 K. Takaishi, M. Yasui and T. Ema, J. Am. Chem. Soc., 2018, 140, 5334-5338.

32 Y. Morisaki, M. Gon, T. Sasamori, N. Tokitoh and Y. Chujo, J. Am. Chem. Soc., 2014, 136, 3350-3353. 
33 E. M. Sanchez-Carnerero, F. Moreno, B. L. Maroto, A. R. O. Agarrabeitia, J. María, B. G. Vo, G. Muller and S. de la Moya, J. Am. Chem. Soc., 2014, 136, 3346-3349.

34 R. B. Alnoman, S. Rihn, D. C. O'Connor, F. A. Black, B. Costello, P. G. Waddell, W. Clegg, R. D. Peacock, W. Herrebout, J. G. Knight and M. J. Hall, Chem.-Eur. J., 2016, 22, 93-96.

35 S. Sato, A. Yoshii, S. Takahashi, S. Furumi, M. Takeuchi and H. Isobe, Proc. Natl. Acad. Sci. U. S. A., 2017, 114, 1309713101.

36 C. Schaffner-Hamann, A. von Zelewsky, A. Barbieri, F. Barigelletti, G. Muller, J. P. Riehl and A. Neels, J. Am. Chem. Soc., 2004, 126, 9339-9348.

37 F. J. Coughlin, M. S. Westrol, K. D. Oyler, N. Byrne, C. Kraml, E. Zysman-Colman, M. S. Lowry and S. Bernhard, Inorg. Chem., 2008, 47, 2039-2048.

38 T.-Y. Li, Y.-M. Jing, X. Liu, Y. Zhao, L. Shi, Z. Tang, Y.-X. Zheng and J.-L. Zuo, Sci. Rep., 2015, 5, 14912.

39 C. Shen, E. Anger, M. Srebro, N. Vanthuyne, K. K. Deol, T. D. Jefferson, G. Muller, J. A. G. Williams, L. Toupet, C. Roussel, J. Autschbach, R. Réau and J. Crassous, Chem. Sci., 2014, 5, 1915-1927.

40 X.-P. Zhang, V. Y. Chang, J. Liu, X.-L. Yang, W. Huang, Y. Li, C.-H. Li, G. Muller and X.-Z. You, Inorg. Chem., 2015, 54, 143152.

41 S. Tanaka, K. Sato, K. Ichida, T. Abe, T. Tsubomura, T. Suzuki and K. Shinozaki, Chem.-Asian J., 2016, 11, 265273.

42 T. R. Schulte, J. J. Holstein, L. Krause, R. Michel, D. Stalke, E. Sakuda, K. Umakoshi, G. Longhi, S. Abbate and G. H. Clever, J. Am. Chem. Soc., 2017, 139, 6863-6866.

43 J. Song, M. Wang, X. Zhou and H. Xiang, Chem.-Eur. J., 2018, 24, 7128-7132.

44 V. W.-W. Yam, K. M.-C. Wong and N. Zhu, J. Am. Chem. Soc., 2002, 124, 6506-6507.

45 B. Ma, J. Li, P. I. Djurovich, M. Yousufuddin, R. Bau and M. E. Thompson, J. Am. Chem. Soc., 2005, 127, 28-29.

46 C. Po, A. Y.-Y. Tam, K. M.-C. Wong and V. W.-W. Yam, J. Am. Chem. Soc., 2011, 133, 12136-12143.

47 C. A. Strassert, C.-H. Chien, M. D. Galvez Lopez, D. Kourkoulos, D. Hertel, K. Meerholz and L. De Cola, Angew. Chem., Int. Ed., 2011, 50, 946-950.

48 M. Krikorian, S. Liu and T. M. Swager, J. Am. Chem. Soc., 2014, 136, 2952-2955.

49 A. Aliprandi, M. Mauro and L. De Cola, Nat. Chem., 2016, 8, 10-15.

50 J. Moussa, K. M.-C. Wong, L.-M. Chamoreau, H. Amouri and V. W.-W. Yam, Dalton Trans., 2007, 3526-3530.

51 H. Sesolis, J. Dubarle-Offner, C. K. M. Chan, E. Puig, G. Gontard, P. Winter, A. L. Cooksy, V. W. W. Yam and H. Amouri, Chem.-Eur. J., 2016, 22, 8032-8037.
52 H. Sesolis, C. K.-M. Chan, G. Gontard, H. L.-K. Fu, V. W.-W. Yam and H. Amouri, Organometallics, 2017, 36, 4794-4801.

53 M. Mauro, A. Aliprandi, D. Septiadi, N. S. Kehr and L. De Cola, Chem. Soc. Rev., 2014, 43, 4144-4166.

54 M. Yoshida and M. Kato, Coord. Chem. Rev., 2018, 355, 101115.

55 V. W.-W. Yam, V. K.-M. Au and S. Y.-L. Leung, Chem. Rev., 2015, 115, 7589-7728.

56 F. N. Castellano and C. E. McCusker, Dalton Trans., 2015, 44, 17906-17910.

57 C.-M. Che and S.-W. Lai, Coord. Chem. Rev., 2005, 249, 12961309.

58 P. Pfeiffer and K. Quehl, Ber. Dtsch. Chem. Ges. B, 1931, 64, 2667-2671.

59 S. Lifson, M. M. Green, C. Andreola and N. C. Peterson, J. Am. Chem. Soc., 1989, 111, 8850-8858.

60 D. Yang, P. Duan, L. Zhang and M. Liu, Nat. Commun., 2017, 8, 15727.

61 F. K.-W. Kong, A. K.-W. Chan, M. Ng, K.-H. Low and V. W.-W. Yam, Angew. Chem., Int. Ed., 2017, 56, 15103-15107.

62 S. Tanaka, K. Sato, K. Ichida, T. Abe, T. Tsubomura, T. Suzuki and K. Shinozaki, Chem.-Asian J., 2016, 11, 265273.

63 T. Ikeda, T. Masuda, T. Hirao, J. Yuasa, H. Tsumatori, T. Kawai and T. Haino, Chem. Commun., 2012, 48, 60256027.

64 H. L.-K. Fu, C. Po, H. He, S. Y.-L. Leung, K. S. Wong and V. W.-W. Yam, Chem.-Eur. J., 2016, 22, 11826-11836.

65 J. Liu, H. Su, L. Meng, Y. Zhao, C. Deng, J. C. Y. Ng, P. Lu, M. Faisal, J. W. Y. Lam, X. Huang, H. Wu, K. S. Wong and B. Z. Tang, Chem. Sci., 2012, 3, 2737-2747.

66 T. Ikeda, M. Takayama, J. Kumar, T. Kawai and T. Haino, Dalton Trans., 2015, 44, 13156-13162.

67 A. Satrijo, S. C. J. Meskers and T. M. Swager, J. Am. Chem. Soc., 2006, 128, 9030-9031.

68 J. Kumar, T. Nakashima, H. Tsumatori and T. Kawai, J. Phys. Chem. Lett., 2014, 5, 316-321.

69 Z. Shen, T. Wang, L. Shi, Z. Tang and M. Liu, Chem. Sci., 2015, 6, 4267-4272.

70 T. Ikeda, K. Hirano and T. Haino, Mater. Chem. Front., 2018, 2, 468-474.

71 Q. Wan, W.-P. To, C. Yang and C.-M. Che, Angew. Chem., Int. Ed., 2018, 57, 3089-3093.

72 T. Kayano, S. Takayasu, K. Sato and K. Shinozaki, Chem.-Eur. J., 2014, 20, 16583-16589.

73 M. M. J. Smulders, M. M. L. Nieuwenhuizen, T. F. A. de Greef, P. van der Schoot, A. P. H. J. Schenning and E. W. Meijer, Chem.-Eur. J., 2010, 16, 362-367.

74 K. Akagi, Chem. Rev., 2009, 109, 5354-5401. 